\title{
Eduarda Moretti
}

Email para correspondência: Eduarda Moretti - fteduardamoretti@gmail.com Fisioterapeuta; Mestre em Fisioterapia pela Universidade Federal de Pernambuco. Professora da Universidade Federal de Pernambuco (UFPE)

RESUMO I A fisioterapia desempenha um papel fundamental entre as opções de tratamento conservador para as disfunçães do assoalho pélvico. Nesse contexto, a telerreabilitação associada ao conceito de mHealth surge como uma importante estratégia por envolver a possibilidade de conduzir uma terapia, avaliação ou treino à distância através de dispositivos móveis. É possível, então, observar dois segmentos de aplicativos móveis estabelecidos: o que disponibiliza uma releitura do tradicional diário miccional e $\circ$ que direciona exercícios para a musculatura do assoalho pélvico. Buscas (outubro/2016) nas principais lojas de aplicativos móveis mostram diversos aplicativos que permitem que - usuário preencha informações diárias a respeito da ingesta de líquido, micções, perdas de urina e utilização de proteção higiênica. São muitos também os que buscam auxiliar a realização de exercícios para $\circ$ assoalho pélvico. Contudo, esses aplicativos são de caráter informativo, mostrando apenas séries de exercícios a serem executadas. Alguns produtos foram lançados no mercado oferecendo sistemas integrados a aplicativos móveis que permitem uma interação em tempo real entre os exercícios do assoalho pélvico realizados pelos usuários e o aplicativo. Apesar de vários aplicativos disponíveis, buscas (outubro/2016) nas principais bases de dados da literatura científica em saúde mostraram a existência de apenas dois registros de estudos referentes ao aplicativo móvel denominado "Tät", cujo objetivo é favorecer o tratamento da incontinência urinária de esforço. A partir de uma análise crítica dos estudos, é possível concluir que existem poucas evidências científicas que suportam a utilização de aplicativos móveis no tratamento de disfunções pélvicas.

Palavras-chaves: Diafragma da pelve; Telerreabilitação; Aplicativos móveis. 
assoalho pélvico é um complexo anatômico formado por diversos ligamentos, músculos e fáscia. Além de fechar a saída da pelve verdadeira separando a cavidade pélvica do períneo, fornece suporte a compartimentos anatômicos vitais, onde encontram-se bexiga, uretra, vagina e útero (mulheres) ou próstata, vesículas seminais e glândulas bulbouretrais (homens), reto e ânus ${ }^{1,2}$. Seus componentes são interligados ${ }^{2}$ e trabalham em conjunto a fim de evitar a incontinência urinária e fecal e o prolapso de órgãos pélvicos durante os aumentos de pressão abdominal e atividades físicas cotidianas. Entretanto, devem permitir a micção e defecação, assim como o parto, no caso das mulheres ${ }^{3}$.

bom funcionamento do assoalho pélvico depende da integridade de seus componentes, bem como de um fluxo sanguíneo e nervoso adequado. Portanto, qualquer alteração em um ou mais componentes desse complexo anatômico-funcional pode gerar alguma disfunção, caso as estruturas íntegras não sejam capazes de suprir o desequilíbrio provocado $^{4,5,6}$.

Otratamento das disfunções do assoalho pélvico envolve intervenções conservadoras e cirúrgicas. A fisioterapia desempenha um papel fund amental entre as opções de tratamento conservador. Entretanto, 0 sucesso fisioterapêutico depende da gravidade da desordem do paciente, da integridade anatômica e nervosa do assoalho pélvico, do tipo e duração do treino, da supervisão, da motivação e da adesão dos pacientes ao tratamento proposto ${ }^{7}$ Como alternativa para melhorar a motivação e adesão de pacientes aos tratamentos, surge a telerreabilitação, cujo princípio envolve a possibilidade de conduzir uma terapia, avaliação ou treino à distância através da tecnologia ${ }^{8-10}$.

Nesse contexto, a utilização de dispositivos móveis, como smartphones e tablets, na área da saúde permite a introdução de um novo conceito: "mobile health" ou mHealth. A Organização Mundial de Saúde define mHealth como a prática de saúde mediada por dispositivos móveis, ou seja, dispositivos sem fio ${ }^{11}$. Muitos aplicativos móveis são desenvolvidos para favorecer a telerreabilitação através desses dispositivos. Quando a questão envolve disfunções pélvicas, é possível observar dois segmentos de aplicativos móveis estabelecidos: o que faz uma releitura do diário miccional e o que direciona suas funções aos exercícios da musculatura do assoalho pélvico.

Buscas (outubro/2016) nas principais lojas de aplicativos móveis Play Store, App Store e Windows Store mostram uma gama de aplicativos com funcionalidades equivalentes as de um diário miccional. Alguns deles são: "Diário Miccional", "EduMicc", "Mictionary", "Daily-P", "Diario micc" e "Day2Day". Todos esses aplicativos permitem que - usuário preencha informações diárias a respeito da ingesta de líquido, micções, perdas de urina e utilização de proteção higiênica. Eles permitem, ainda, que após o período de preenchimento do diário, seja enviado um relatório ao terapeuta responsável através do correio eletrônico.

Nesse cenário, o aplicativo móvel EduMicc destaca-se por disponibilizar também informações educacionais importantes e uma plataforma na web para que os profissionais de saúde possam se conectar a seus pacientes. Assim, além das funcionalidades comuns aos demais aplicativos, o EduMicc possibilita uma interação entre terapeuta e paciente, na qual o terapeuta é sincronizado com as atualizações de seus pacientes e recebe os registros em tempo real. Por meio dessa plataforma da web, o terapeuta pode, ainda, enviar alarmes individualizados com orientações para que seus pacientes possam se lembrar de ingerir líquido ou ir ao banheiro, por exemplo.

Quanto aos aplicativos que apresentam funções para auxiliar o treinamento da musculatura do assoalho pélvico, as pesquisas (outubro/2016) nas principais lojas revelaram a existência de diversas opções como "Kegel Exercises", "Kegel Trainer", "MaxKegel", "Pelvic Floor and Kegel Exercises", "EjerciciosKegel" e "Kegel Bootcamp". Contudo, esses aplicativos são de caráter informativo, pois mostram apenas séries de exercícios a serem executadas, sem feedback fidedigno do que o paciente realiza e sem o controle da qualidade na forma de execução.

Diante dessa lacuna, alguns produtos foram lançados no mercado oferecendo sistemas integrados a aplicativos móveis que permitem uma interação em tempo real entre os exercícios do assoalho pélvico realizados pelos usuários e o aplicativo. Denominados Kgoal, Elvie, Skea - Smart 
Kegel Exercise Aid e Magic Kegel, esses são alguns dos sistemas disponíveis no mercado que resultam de uma integração entre hardware e software e são compostos por um dispositivo de silicone para introdução no canal vaginal e um aplicativo móvel. O design desses dispositivos de silicone é semelhante e todos possuem um sensor pressórico, com Bluetooth ${ }^{\circledR}$ integrado, que envia as informações captadas da musculatura do assoalho pélvico para os respectivos aplicativos móveis. Cada aplicativo disponibiliza programas de exercícios a serem realizados através de jogos ou gráficos interativos, que possuem características variadas de cenários e objetivos. Contudo, o conteúdo disponível em relação a esses dispositivos ainda é bastante limitado, pois a maioria das informações acessíveis são de caráter comercial. Não existem, portanto, evidências de que os protocolos estabelecidos nesses aplicativos respeitam as particularidades fisiológicas e biomecânicas dos músculos do assoalho pélvico, nem as características individuais dos usuários.

Apesar de vários aplicativos disponíveis, buscas (outubro/2016) nas principais bases de dados da literatura científica em saúde (MEDLINE/PubMed, CINAHL, CENTRAL, LILACS) mostraram a existência de apenas dois registros ${ }^{12,13}$ referentes a um mesmo aplicativo móvel denominado "Tät", desenvolvido na Suécia por uma equipe da Umeå University. Esse aplicativo foi desenvolvido com a finalidade de favorecer o tratamento da incontinência urinária de esforço e fornece informações e orientações sobre incontinência urinária e hábitos de vida, além de instruções para realização de um programa de exercícios para a musculatura do assoalho pélvico que envolve comandos visuais e sonoros e a possibilidade de programar lembretes para realização do treinamento proposto.

Um estudo foi, então, conduzido a fim de avaliar a efetividade da utilização do Tät para o tratamento para incontinência urinária de esforço. Participaram do estudo 123 mulheres entre 18 e 72 anos, alocadas em dois grupos: um grupo que utilizou 0 aplicativo por três meses e outro grupo que ficou sem intervenções, tendo acesso ao aplicativo após os três meses. Foram avaliadas a gravidade dos sintomas da incontinência urinária, a qualidade de vida das voluntárias, a frequência de episódios de incontinência por semana e a percepção de melhora das participantes. Os resultados das análises entre os grupos mostraram melhorias estatisticamente significantes e clinicamente relevantes em relação a esses desfechos, favorecendo o grupo que utilizou o Tät. Além disso, $66,7 \%$ das mulheres mostraram-se satisfeitas com o aplicativo, $96,7 \%$ das participantes o classificou como "bom" ou "muito bom" e 100\% da amostra do grupo experimental recomendou sua utilização. Nesse sentido, foi proposto pelos autores do estudo que o tratamento para mulheres com incontinência urinária de esforço não precisa ser acompanhado presencialmente e pode ser conduzido através de aplicações móveis, inclusive através do aplicativo Tät, que se mostrou uma alternativa efetiva e de fácil acesso ${ }^{12,13}$.

Os resultados com o aplicativo sueco foram bastante interessantes, entretanto, para incorporá-los à clínica, deve-se considerar questões que implicam na aplicabilidade prática desses resultados. São elas:

a) existe semelhança entre o tipo de participante do estudo, a intervenção proposta e a comparação com o cenário clínico no qual estamos inseridos?

b) a intervenção estudada está disponível e é viável em nosso ambiente de trabalho?

c) qual a relação entre os potenciais benefícios e malefícios da terapia em questão?

d) quais as expectativas e perspectivas dos nossos pacientes?

e) quais as barreiras e os facilitadores para a implementação da intervenção no contexto de vida do nosso paciente?

Essas questões ajudam na interpretação dos achados dos estudos dentro do contexto da prática clínica e são facilmente acessadas através do aplicativo móvel CrivoApp, desenvolvido com o objetivo auxiliar a leitura crítica de artigos científicos na área de saúde.

Diante do exposto e após análise crítica dos artigos referentes ao Tät, pode-se concluir que, apesar de muitos aplicativos móveis disponíveis, existem poucas evidências científicas que suportam a efetividade de sua utilização no tratamento de disfunções pélvicas. Ademais, essas evidências tornam-se bastante limitadas quando é considerada a prática clínica no Brasil, uma vez que existe uma diferença sociocultural importante entre Brasil e Suécia. 
Contudo, a telerreabilitação através de aplicativos móveis é uma alternativa em potencial para o tratamento das desordens do assoalho pélvico e esforços em pesquisas científicas são essenciais para que achados mais concretos possam fundamentar uma prática clínica baseada em evidências.

\section{CONFLITOS DE INTERESSES}

Nenhum conflito financeiro, legal ou político envolvendo terceiros (governo, empresas e fundações privadas, etc.) foi declarado para nenhum aspecto do trabalho submetido (incluindo mas não limitandose a subvenções e financiamentos, conselho consultivo, desenho de estudo, preparação de manuscrito, análise estatística, etc). Entretanto, a autora reportou participação na criação do EduMicc e do CrivoApp.

\section{REFERÊNCIAS}

1. Lalwani N, Moshiri M, Lee JH, Bhargava P, Dighe MK. Magnetic Resonance Imaging of Pelvic Floor Dysfunction. Radiol Clin North Am. Elsevier Inc; 2013;51 (6):1 1 27-39. doi: 10.1016/i.rcl.2013.07.004

2. Stein TA, DeLancey JOL. Structure of the perineal membrane in females: gross and microscopic anatomy. Obstet Gynecol. 2008;1 11 (3):686-93. doi: 10.1097/ AOG.0b013e318163a9a5

3. Ashton-Miller JA, DeLancey JOL. Functional anatomy of the female pelvic floor. Ann N Y Acad Sci. 2007;1 101:266-96. doi: 10.1196/annals.1389.034

4. Memon $\mathrm{H}$, Handa VL. Pelvic floor disorders following vaginal or cesarean delivery. Curr Opin Obstet Gynecol. 2012;24(5):349-54. doi: $10.1097 /$ GCO.0b013e328357628b

5. Shah AP, Mevcha A, Wilby D, Alatsatianos A, Hardman JC, Jacques $S$ et al. Continence and micturition: an anatomical basis. Clin Anat. 2014;27(8):1275-83. doi: 10.1002/ ca. 22388

6. Stoker J. Anorectal and pelvic floor anatomy. Best Pract Res Clin Gastroenterol. 2009;23(4):463-75. doi: 10.1016/i. bpg.2009.04.008

7. Ferreira M, Santos P. Evidência cientifica baseada nos programas de treino dos músculos do pavimento pélvico. Revista de Exemplo. 2011:309-18

8. Spinardi $A C P$, Blasca WQ, Wen CL, Maximino LP. Telefonoaudiologia: ciência e tecnologia em saúde. Pró-Fono Rev Atualização Científica. Pró-Fono Departamento Editorial.
$2009 ; 21(3): 249-54$

9. McCue M, Fairman A, Pramuka M. Enhancing quality of life through telerehabilitation. Phys Med Rehabil Clin N Am. 2010;21(1):195-205. doi: 10.1016/i.pmr.2009.07.005

10. dos Santos MTN, Moura SCDO, Gomes LMX, Lima AH, Moreira RS, Silva CD et al. Telehealth application on the rehabilitation of children and adolescents. Rev Paul Pediatr orgão Of da Soc Pediatr São Paulo. 2014;32(1):136-43

1 1. Kay M. mHealth: New Horizons for Health through Mobile Technologies. World Heal Organ. 2011 ;3:66-71

12. Asklund I, Nyström E, Sjöström M, Stenlund H, Samuelsson E. Treatment of stress urinary incontinence via a smartphone application : a randomised controlled trial. Int Cont Soc Annu Meet; 2014

13. Nystrom E, Asklund I, Sjostrom M. High expectations for pelvic floor muscle training with mobile application predicts successful treatmente in women with stress urinary incontinence. Neurourology and urodynamics. 2015;34(3):S448-449 